\title{
Rate Control Optimization Algorithm of Ubiquitous Power Sensor Network Tester Based on Multimedia
}

\author{
Bing Lu, Miao Jin (iD, Xiwen Chen, Wei Zhou, Gaoning Nie, and Quan Wang
}

China Electric Power Research Institute, Wuhan 430074, China

Correspondence should be addressed to Miao Jin; jinmiao@epri.sgcc.com.cn

Received 7 December 2021; Revised 4 January 2022; Accepted 15 January 2022; Published 12 February 2022

Academic Editor: Haibin Lv

Copyright ( 92022 Bing Lu et al. This is an open access article distributed under the Creative Commons Attribution License, which permits unrestricted use, distribution, and reproduction in any medium, provided the original work is properly cited.

\begin{abstract}
With more and more video application scenarios and increasing complexity, the popularization of multimedia information and the urgent need to update wireless sensor networks have led to the emergence and development of wireless multimedia sensor networks. This paper focuses on the rate control optimization algorithm of multimedia-based ubiquitous power sensor network, which is mainly studied from three aspects: constructing algorithm verification platform based on multimedia technology, performance analysis, and optimization of rate control optimization algorithm based on multimedia technology. An algorithm verification platform is established based on multimedia technology. This article uses a scalable video coding method to design a client-driven scalable video transmission system. The simulation results show that the algorithm can effectively improve the video quality. The algorithm of rate control based on multimedia technology is proposed. A reliable multipath routing algorithm based on AODV is proposed. Based on the energy balance algorithm, the node-independent routing mechanism and link quality estimation model are introduced. An independent and reliable active/standby communication link to the node of the destination node is established. Linux, the full name of GNU/Linux, is a free-to-use and freely disseminated Unix-like operating system. It is a POSIX-based multiuser, multitasking, multithreaded, and multi-CPU operating system. With the development of the Internet, Linux has gained support from software enthusiasts, organizations, and companies all over the world. In addition to maintaining a strong momentum of development in servers, it has made considerable progress in personal computers and embedded systems. Under the Linux platform, a large number of tests are performed and the performance of the control code rate is analyzed. The socalled sensor network is a distributed intelligent network system that is composed of a large number of small sensor nodes with wireless communication and computing capabilities deployed in the active area through a self-organizing method that can autonomously complete designated tasks according to the environment. The distance between the nodes of the sensor network is very short, and the multihop wireless communication method is generally used for communication. The sensor network can run in an independent environment, or it can be connected to the Internet through a gateway so that users can access it remotely. It is concluded that $\mathrm{ABR}$ is the most suitable rate control algorithm for video transmission. According to the CQP experiment results, the QP value set in the improved ABR algorithm is at most 40; the security from the construction of the ubiquitous power sensor network to the final monitoring data collection and upload process is verified by simulating the hypothesis attacks and faults. It is proved that the construction of ubiquitous power sensor network can reduce the energy consumption and delay of monitoring data collection and verify the superiority of constructing ubiquitous power sensor network.
\end{abstract}

\section{Introduction}

Wireless video transmission refers to a monitoring system that uses radio waves to transmit video, sound, data, and other signals without wiring (cables). There are two main concepts for wireless image transmission, that is, real-time video transmission. One is transmission on the move, that is, mobile communication, and the other is broadband transmission, that is, broadband communication. In wireless video transmission, because of the limitation of transmission bandwidth, it is necessary to control the transmission bit rate of the video. This technology is called bit rate control. Its purpose is to ensure the smooth transmission of the compressed bit stream in the channel and the reasonable 
utilization of the effective bandwidth. According to the positive or negative difference between the output coding bit rate of the encoded frame and the target bit rate, it can be judged whether the coding bit rate at the moment is too large or too small. The joint adjustment of these two points makes the bit rate control more accurate. To some extent, the test system has shifted from research and development to a complex production process. Simplifying the whole process and ensuring the completeness and continuity of the test after the transformation has also become one of the important research directions. Bit rate is also called "binary bit rate," commonly known as "bit rate," indicating the number of transmitted bits per unit time. It is used to measure the transmission speed of digital information, often written as bit/sec. According to the number of bits occupied by each frame of image storage and the transmission bit rate, the transmission speed of digital image information can be calculated.

In a computer system, multimedia refers to a humancomputer interactive information exchange and dissemination medium that combines two or more media. The media used include text, pictures, photos, sounds, animations and videos, as well as interactive functions provided by the program. With the continuous development of the Internet and the continuous popularization of multimedia information technology, human beings have higher and higher requirements for information, and the demand is more and more abundant, while the traditional ubiquitous power sensor network system collects less multimedia information. There are several types of multimedia information, such as video, voice, images, text, and so on. Among them, video information is popular because of its convenience, content diversification, convenient storage, processing, and so on. With the rapid development of sensor technology, such as CMOS (Complementary Metal Oxide Semiconductor) camera, video collection becomes more and more convenient. Therefore, as video application scenarios become more and more complex and complex, the popularity of multimedia information and the urgent need to update the sensor network lead to the emergence and development of ubiquitous power sensor networks.

Based on the practicability of ubiquitous networks, many research teams at home and abroad have conducted in-depth research. Park et al. [1] found that the uneven energy consumption caused by the integrated routing sensor of the topology deployment type is a factor that reduces the quality of service (QoS) and the lifetime of the overall topology with respect to the user's convenience service. Gope [2] proposed a new mutual authentication and key agreement scheme and resulted in significantly less computational overhead than others' schemes. Santana et al.'s [3] strategy promotes wireless interface deactivation between nodes that do not participate in any routing, which helps to save a lot of power for the grid. The strategy they studied was conducive to wireless transmission, which meant that it preferentially deactivated nodes that received interference from other parts of the smart city. Ubiquitous networks are literally widespread. The ubiquitous network means that people are placed in a ubiquitous network, meaning that computers are integrated into people's living spaces, forming an "everywhere, everywhere" invisible "network environment" $[4,5]$. Chai and $\mathrm{Xu}$ [6] aimed at strengthening the production management of traditional workshops, strengthening the workshop production management, improving the production management level of the workshop, and researching the construction of smart workshops based on ubiquitous network technology. $\mathrm{Ni}$ et al. [7] established an integrated information service security policy management architecture for digital libraries for ubiquitous networks by in-depth study of big data, cloud computing, mobile Internet technology, and user service requirements. The development of ubiquitous networks will change the content and mode of information organization and conduct in-depth research on the mechanism of information organization in the new network environment [8]. Duan and Liu [9] studied the maturity of government information services in the ubiquitous network environment and can effectively evaluate government information services under the ubiquitous network environment. Pan and Chen [10] and others provided some reference for the innovation of library smart service mode and the improvement of library service level under the background of ubiquitous network. With the continuous development of network technology, the new network learning mode, that is, ubiquitous learning, has received more attention and development, and the introduction of ubiquitous learning into experimental teaching will be the inevitable result of future educational development [11]. Zhang [12] analyzed the concept of knowledge fusion in the ubiquitous network environment, and discussed the related technologies of ontology technology, semantic web, data mining and other knowledge fusion. Zhuoma [13] proposed that ubiquitous network construction requires the participation of social enterprises. It is necessary to adopt special policies to open existing public facilities' optical fiber lines and to promote the construction of smart communities on the basis of ubiquitous networks. Many research teams have used many research methods for ubiquitous network research, but their methods have certain drawbacks. The rate control algorithm has gradually become a research topic because of its simplicity, convenience, high operability, and high fault tolerance.

Nowadays, the rate control algorithm has been widely studied. In order to make the network transmission smoother and more stable, the researchers have done a detailed and rigorous research on the problems in the rate control and applied it in various fields. Etesaminia and Mazinan [14] apply the rate control algorithm to image processing. The proposed algorithm encodes only the coding channels contained in the final code stream and does not require any postcompression rate control. Park et al. [15] applied the rate control algorithm to the research of mobile devices and proposed a new coding rate control algorithm for uplink video services based on the estimated network conditions. Lakshmi et al. [16] applied the rate control algorithm to the network deployment. It was found that the flow control of data size and rate maximizes throughput and packet transmission with low power consumption. Guo et al. $[17,18]$ proposed that rate control is an important module in 
the video coding system, which is used to control the code rate of the encoder to output a specific target and minimize the distortion of the encoded video; in order to meet the accuracy of the code rate control to improve the encoder rate distortion performance, a frame rate control algorithm based on improved $R-\lambda$ model is proposed. The average bit rate relative error is $0.095 \%$; the average bit rate savings (BDRate) is $2.6 \%$ based on HM16.7 with no code rate control. Yan et al. [19] proposed an MV-HEVC rate control algorithm based on similarity analysis for the problem that the current MV-HEVC has not given effective rate control. Zhan et al. [20] applied the rate control algorithm to video playback and proposed a slice-level rate control algorithm. Experimental results show that the algorithm can accurately control the slice rate and ensure video quality. Tang et al. [21] proposed an improved HEVC code rate control based on content complexity for high-efficiency video coding (HEVC) using average absolute error in rate control to inaccurate image complexity estimation and bit allocation deviation. Liu et al. [22] applied the rate control algorithm in scene switching. The experimental results show that compared with HEVC's own rate control algorithm, this strategy can effectively reduce the negative impact of scene switching. Chen et al. [23] proposed a complexity-based HEVC intraframe rate control algorithm. The proposed method can make the output code rate closer to the target bit rate and further improve the video quality. Zheng et al. [24] reported that for the existing interpolation structure without feedback distributed video compression (DVC) under the condition of large image group, there is a serious decoding delay problem, and the feedback-free DVC system of extrapolation structure is studied. Rong and Yang [25] proposed a pixel-based linear weighted histogram for the inaccuracy of image complexity estimation and the irrationality of bit allocation in the LCU layer using the average absolute error (MAD) in the HEVC rate control scheme. Link quality is one of the research hotspots in the field of microgrid communication wireless sensor networks. The nonlinear and nonstationary random characteristics of wireless link quality are difficult to achieve accurate prediction and control of its reliability.

The structure of the confidence interval prediction algorithm for the reliability of wireless communication links is proposed, and the approximate decoupling algorithm of the link quality represented by the signal-to-noise ratio and the confidence interval prediction algorithm of the link quality based on the wavelet neural network in the algorithm structure are studied, respectively. In order to solve the problem of complex operation and low accuracy in ubiquitous power sensor networks, this paper focuses on three aspects of the rate control optimization algorithm for multimedia-based ubiquitous power sensor networks: building multimedia-based technologies. These three aspects are from the algorithm verification platform, the performance analysis of the rate control optimization algorithm based on multimedia technology, and the performance analysis of the ubiquitous power sensor network. A reliable multipath routing algorithm based on AODV is proposed. The nodeindependent routing mechanism and link quality estimation model are introduced to verify the security from the construction of ubiquitous power sensor network to the final monitoring data collection and uploading process. The code based on multimedia technology is proposed. The rate control optimization algorithm, under the Linux platform, carries out a large number of tests and analyzes its control code rate performance, and it is concluded that $A B R$ is the most suitable rate control algorithm for video transmission and constructs a ubiquitous power sensor by analyzing and comparing the energy consumption and delay in the case of conventional networks and ubiquitous power sensor networks. Multimedia technology is a technology that uses computers to store and manage various information such as language, data, audio, and video so that users can communicate real-time information with computers through multiple senses. The content displayed and carried by multimedia technology is actually the product of computer technology.

\section{Method}

2.1. Ubiquitous Network System. As telecommunications services shift from voice-based to data-based, switching technology has also gradually shifted from traditional circuit switching technology to packet-based data switching and broadband switching, adapting to the integrated features of IP-based services in next-generation networks. The transmission of information is no longer as singular as the previous voice communication and written communication. The full development of optoelectronic technology has made the transmission of information more diversified; the processing of information used to rely mainly on human brain judgments, while computer technology in modern society telling development has increased the processing speed and processing accuracy of information and can accomplish some things that humans cannot accomplish. At present, the ubiquitous network has been regarded as the direction of the development and evolution of information and communication networks. For the implementation and research of ubiquitous networks, many countries and regions have put forward the concept of ubiquitous services and corresponding national strategies from the perspective of long-term development so as to seize the commanding heights of network strategies. As one of the most important and basic tools and technologies for collecting information on the physical world, sensor networks will surely play a very important role in the process of deploying ubiquitous networks as an absolute main member of the extension layer of the ubiquitous network architecture. An important bridge between people and the environment. The generalized network system architecture is shown in Figure 1.

The architecture of the ubiquitous network system can be divided into two layers: application and network. The network is the carrier of the application, and the application is built on the network. "Network service" refers to some service-oriented software modules based on distributed programs that run on the network. Network services use common Internet standards such as HTTP and XML (a 


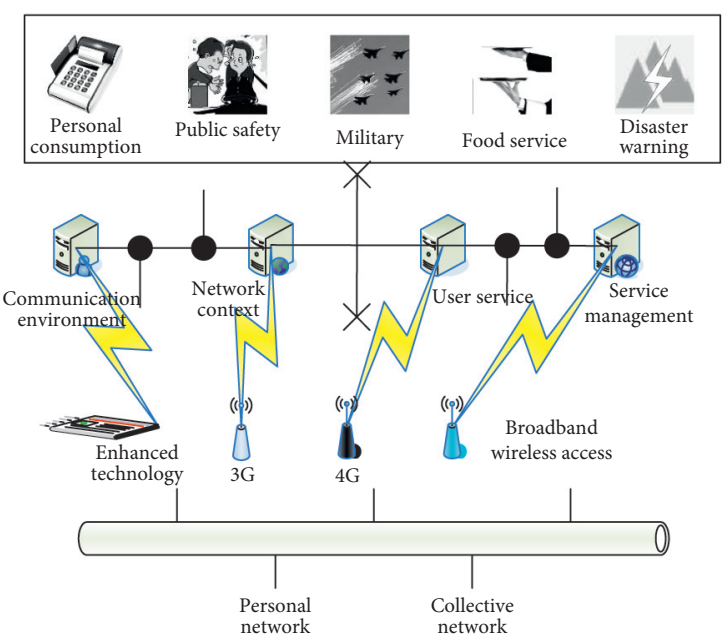

FIgURE 1: Ubiquitous network architecture. subset of the standard universal markup language) to enable people to access WEB data in different places through different terminal devices.

\subsection{Ubiquitous Power Sensor Network Reliable Multipath} Routing Algorithm. Based on the proposed energy equalization algorithm, a node-independent routing mechanism and a link quality estimation model are introduced to establish an independent and reliable active/standby communication link to the destination node. First, the primary path is used to transmit data. When the primary path is interrupted or the data transmission fails due to data congestion, the source node switches to the alternate path to transmit data; when the standby link fails, it enters the route maintenance phase, and the source node uses the redundant relay node to construct a weak ring topology to complete data communication and achieve reliable data transmission. The basic block diagram of the algorithm is shown in Figure 2.

Microgrid is a regional controllable basic micro power generation and distribution system composed of controllable distributed power sources, energy storage, load power networks, and communication control networks connected together. It can operate in island mode without the power grid, or it is connected to the grid and runs in grid-connected mode. In order to extend the life of the node, minimize the working time of the microprocessor, especially when there is no information analysis and processing, the microprocessor and the entire node are in a dormant state to increase the life of the sensor node. The energy supply module can maintain the normal operation of the sensor node software and hardware system, and the energy supply module requires stable working voltage and working current. This structure is shown in Figure 3.

2.2.1. Estimation of Loss Rate. Data compression technology is mainly used to reduce the size of video data to meet the needs of storage and transmission, but the existing image processing technology must first fully decompress the video compression stream before processing.

The link quality of the periodic estimator is to add a monotonically increasing sequence number in the sensor node data, and the receiving node estimates the unidirectional link quality between the nodes according to the received data information statistical packet loss situation. Assume that the number of data packets received by node $i$ in the last node is $n_{\text {rev }}$, the number of lost data is $n_{\text {loss }}$, and smoothing coefficient is $\lambda \in[0,1]$; then the data receiving success rate of node $i$ during this time is estimated as follows:

$$
\begin{aligned}
& r_{i}=\lambda \times r_{i}+(1-\lambda) \times \frac{n_{\mathrm{rov}}}{n_{\mathrm{rev}}+n_{\mathrm{loss}}}, \\
& r_{i}=r_{i} \otimes \frac{\left(\lambda \cdot n_{i}^{2}\right)}{\left(\sum_{r=1}^{n-1} \lambda \cdot n_{r}^{2}\right)} .
\end{aligned}
$$

2.2.2. Link Reliability. As a new generation of wireless communication technology, wireless sensor networks have unique advantages such as low power consumption and selforganization, which are considered to have broad application prospects in the fields of data acquisition, operation monitoring, fault diagnosis, and remote control of microgrids.

A new link performance model is established by defining link reliability to measure link reliability performance. The reliability $P_{r}$ of the link formed by the $k$ sensor nodes on the path from the source node to the destination node can be calculated by

$$
\begin{aligned}
& P_{r}=\prod_{i=1}^{k} r_{i}, \\
& p_{r}=\lambda(i-r)_{i-1} \cdot \frac{1}{p-1}+(i-r)_{i} * \frac{1}{r(p-1)} .
\end{aligned}
$$

2.2.3. Reliable Link Quality Model. Let the number of hops from the source node to the destination node be $N$, the remaining energy of node $i$ on the link be $E_{i}$, and the node load be $L_{i}$; then the performance function of the link based on link reliability is defined as shown in the following equation.

$$
F_{r}(P)=\frac{P_{r} \times \sum_{i=1}^{N}\left(P_{i} / P_{\max }\right)}{\left(N \times \sigma_{E}\right)} .
$$

2.2.4. Entropy Weight Coefficient Method. When the value of an attribute value on a different scheme is closer to its entropy value, the entropy $E_{k}$ of the attribute $k$ is larger, indicating that the closer the value of the attribute is to each scheme, the optimal value of the attribute value, and the attribute. The smaller the difference between the values, the closer to the optimal value. The attribute value in each neighbor node is calculated by the following formula, and 


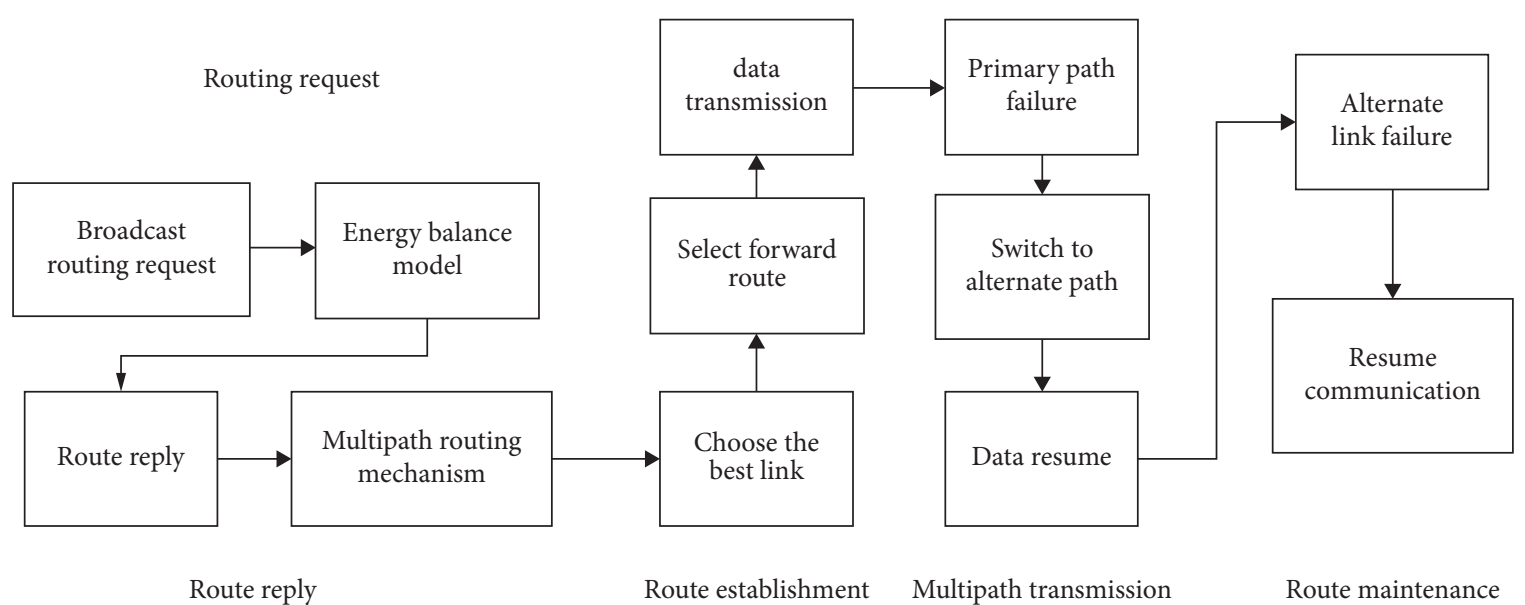

FIgURE 2: Algorithm basic frame diagram.

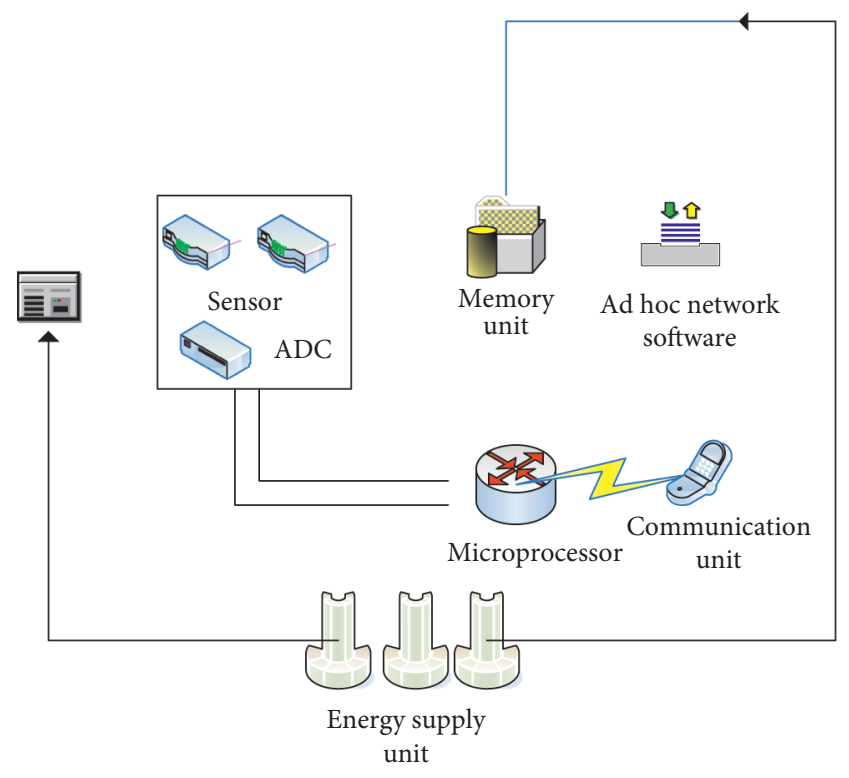

FIGURE 3: Sensor network node structure.

the probability $p_{i}^{k}$ of the $N_{i}$ attribute $k$ of the neighbor node is obtained as follows:

$$
\begin{aligned}
p_{i}^{k} & =\frac{N_{i k}}{\sum_{i=1}^{m} N_{i k}} \\
E_{k} & =-\sum_{i=1}^{m}\left(\frac{N_{i k}}{\sum_{i=1}^{m} N_{i k}}\right) \log \left(\frac{N_{i k}}{\sum_{i=1}^{m} N_{i k}}\right) .
\end{aligned}
$$

2.2.5. Weight Calculation. Since there is a certain attribute value difference between the same attribute values between neighboring nodes, the smaller the difference is, the closer the value of the attribute is, and the smaller the effect on the decision is, the smaller the corresponding weight should be given, and the weight given by the opposite. It should be bigger.

$$
\begin{aligned}
& R_{k}=\frac{E_{k}}{\log (m)}, \\
& w_{k}=\frac{\left(1-R_{k}\right)}{\left(l-\sum_{k=1}^{I} R_{k}\right)} .
\end{aligned}
$$

$w_{k}$ satisfies $0<w_{k}<1, \sum_{k=1}^{l} w_{k}=1$, and the obtained weight is substituted into the energy balance model to obtain the energy balance of each node in the neighborhood $A=1$, and the node with better performance is selected by the energy balance control to forward the routing request.

\subsection{Rate Control Algorithm Based on Ubiquitous Grid Sensor} Network. The H.264 standard rate control scheme JVTG012 uses the Lagrangian function to control the video compression rate. The following formula lists the rate distortion cost function of the H.264 standard:

$$
J=\mathrm{SATD}+\lambda * R,
$$

$$
p^{1-\lambda}=\frac{\sum_{i=1}^{0} r\left(p_{r=1}\right) \cdot\left(j\left(\|i-j\|^{2}\right)\right)^{i-r}}{\prod j_{1,2, \ldots, i}} .
$$

$J$ represents the rate distortion generation value, $R$ is the minimum number of coded bits, $\lambda$ represents the rate distortion cost coefficient, SATD represents the absolute error sum, and the time domain difference of the coded residual can be calculated, which also affects the value of the peak signal-to-noise ratio (PSNR). The image complexity of the current frame is predicted based on the SATD value, and the image complexity is calculated by weighting the SATD value of the encoded frame and the SATD value of the current frame.

$$
\text { Blurred }- \text { complexity }_{i}=\frac{\sum_{n=1}^{i} \operatorname{SATD}(n) *(0.5)^{i-n}}{\left(\sum_{n=1}^{i}(0.5)^{i-n}\right)} .
$$

After predicting the complexity of the current coded frame, the following code rate control empirical formula 
is used to derive the quantization coefficient qpscale corresponding to the current frame.

$$
\text { qpscale }=\text { Complexity }{ }^{1-q c o m p} \text {, }
$$

qcomp denotes a linear quantization control parameter, generally taking two values. When qcomp $=0.0$, it indicates a fixed code rate value; when qcomp $=1.0$, it indicates a fixed quantization value. After obtaining the quantized coefficients of the current encoded frame, X264 will divide the quantization coefficient qpscale of the current coded frame into quarters based on the number of bits generated by the previous coded frame.

The current coded frame quantization coefficient is updated to the following mediation formula:

$$
\text { qpscale }=\frac{\text { qpscale }}{\text { rate_factor }} \text {. }
$$

The final quantization parameter is obtained by the following formula. According to the above formula, when qpscale is smaller, the smaller the $\mathrm{qp}$ is, the smaller the quantization parameter is, and the purpose of increasing the code rate is achieved; on the contrary, when qpscale is larger, qp is increased while the code rate is decreased.

$$
\mathrm{qp}=\alpha+\beta \log _{2}\left(\frac{\mathrm{qpscale}}{\gamma}\right) \text {, }
$$

where $\alpha=12, \beta=6$, and $\gamma=0.85$ are empirical coefficients.

\subsection{Media Adaptive Technology}

2.4.1. Online Adaptive Transcoding. The online transcoding of the media content is that the compressed video stream passes through a transcoder before being transmitted to the client, and the original stream is converted into another different one by dynamically changing the encoding parameters, the frame rate, the spatial resolution, and the like. A schematic diagram of online transcoding is shown in Figure 4.

The online transcoding can enhance the adaptability of the media, support heterogeneous networks and terminals, and support different systems and different formats. Online transcoding can enhance the adaptability of media, support heterogeneous networks and terminals, and support different systems and different formats. The analysis of the experimental results of the target code rate for different sequences is shown in Table 1.

2.4.2. Scalable Video Coding (SVC). SVC's temporal scalability refers to the ability to extract code streams of different frame rates from a single video stream. T0 denotes a base layer, and T1, T2, and T3 represent enhancement layers. In interprediction, the frames formed by the layers of $\mathrm{T} 1$ and $\mathrm{T} 2$ refer only to the frames in the layer, and the frames in the T3 layer need to refer to the frames in the T1 or T2 layer. When extracting a frame containing only $\mathrm{T} 0$, that is, a base layer code stream, the frame rate is $3.75 \mathrm{fps}$ at this time; that is, the video is played at a speed of 3.75 frames per second. When a frame containing $\mathrm{T} 1$ and $\mathrm{T} 2$ layers is extracted, the video frame rate reaches $7.5 \mathrm{fps}$. Similarly, the frames of the T0, T1, and T2 layers are extracted, and the video frame rate is 15 fps. When all four layers of frames are extracted, $30 \mathrm{fps}$ will be completely restored. Different frame structures correspond to different extraction methods.

Two quality layering methods are defined in SVC: coarse grain quality scalability (CGS) and medium grain quality scalability (MGS). The basic idea is to obtain differentquality images by controlling the quantization parameters in the interlayer prediction of quality stratification; that is, by adopting a smaller quantization step, the high-quality layer can obtain finer and higher quality than the low-quality layer. The difference is that the CGS-coded transform coefficients are fixed, and the MGS-coded transform coefficients can be intercepted at any point.

2.5. Multimedia B/S Architecture. Here, the Browser/Server architecture belongs to a three-layer structure, and its threelevel structure is shown in Figure 5. In the B/S structure, the first-level browser is the presentation layer, and the main function is to implement the user. The interface is fully docked. When the client sends a specific request to the web server specified by the URL (Uniform Resource Locator), the web server transmits the corresponding file data to the user by using the HTTP protocol, and the client can display the WWW-based browser after accepting.

The second layer of the Web server is a functional layer; the main function is to achieve the customer's specific application; that is, when the Web server accepts the client request, it will use the CGI program and ASP or JDBCODBC interface and the database is docked to implement the application processing, after which the results of the database are returned to the web server and then transferred to the corresponding client. As for the third-tier database server, it is the data layer. The main function is to implement independent data processing in response to the client's request. The Browser in the Browser/Server system is a type of general-purpose browser. It does not have any application in the normal sense. The middle layer in the Browser/Server system is a very important core part of the $\mathrm{B} / \mathrm{S}$ structure.

\subsection{NoSQL Database}

2.6.1. Key-Value Storage. A key-value database is essentially a hash table, similar to the data structure used by the HashTable collection in the Java language. Stored according to the key value, the value corresponding to each key value can be an arbitrary value. NoSQL does not impose too many restrictions on value and can be freely determined by each product. In general, NoSQL databases only provide simple operations such as set, get, and delete based on the key value, and do not provide operations for a value's value.

2.6.2. Document Storage. The design of the document type database is inspired by the Lotus Notes office software, which is also stored based on key, but the corresponding value is no 


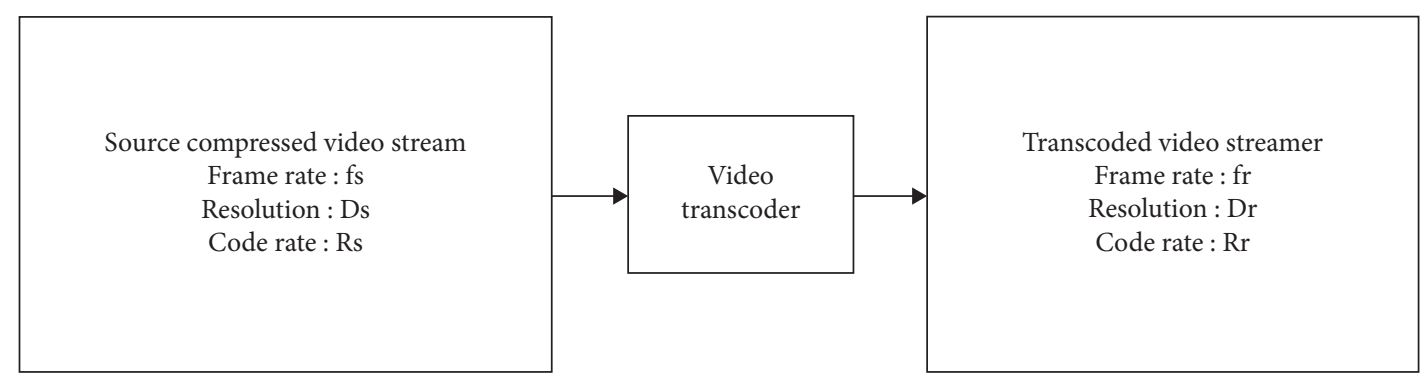

FIgURE 4: Online transcoding diagram.

TABLE 1: Sequence experiment results.

\begin{tabular}{cccc}
\hline Sequence & Target bit rate & The actual bit rate of the original algorithm & Average value \\
\hline & 100 & 98.13 & 32.44 \\
A & 200 & 197.42 & 36.52 \\
& 500 & 503.17 & 39.46 \\
& 800 & 821.88 & 41.02 \\
\hline & 100 & 97.21 & 34.58 \\
B & 500 & 196.87 & 37.23 \\
& 500 & 496.97 & 39.91 \\
& & 899.01 & 42.59 \\
\hline
\end{tabular}

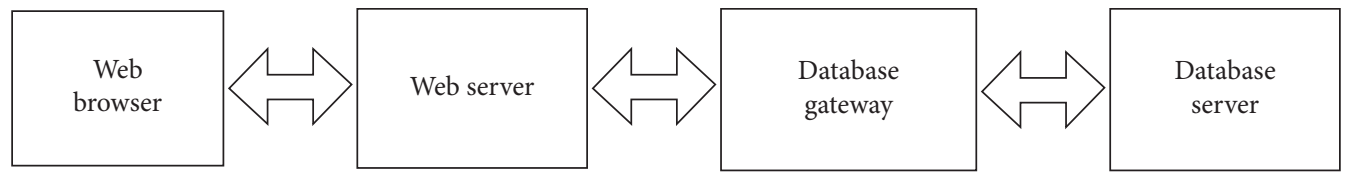

Figure 5: B/S architecture mode diagram.

longer an arbitrary value, but a structured document. This structured document must meet specific storage formats such as XML, JSON, and more. A document-based database can be thought of as an upgraded version of a key-value database that allows nested key values within a document to implement complex storage.

2.6.3. Column Storage. Column storage relies on a table model in a relational database, but it does not provide a multitable join operation like Join in a relational model. The design idea is that when the user stores the data, the "Column" is used as the storage focus point, which is significantly different from the "Row" in the relational database. From the perspective of physical storage, since the minimum storage unit of the hard disk is the page, the relational database stores the data of the same record in the table in the same page of the hard disk, and the column storage stores the data belonging to the same column.

2.6.4. Graph Storage. The graph database uses graph structure correlation algorithms to implement data storage, such as shortest path addressing algorithm and $N$ degree relationship lookup. When using the graph data model, it is often necessary to calculate the entire graph to get the required information. The calculation is relatively complicated, and the graph structure is not suitable for the distributed cluster scheme. The graphical database is more suitable for social networks, recommendation systems, and so on, focusing on building relational graphs. Comparison of different storage rules and related parameters is shown in Table 2.

\section{Experiment}

3.1. Experimental Platform and Equipment. Arm-based Linux video acquisition is mainly used for the acquisition of video image sequences, which is to prepare for the following modules such as video encoding. This article mainly implements ARM9 acquisition based on arm9. The selected USB camera model is WD-HA603F; the picture below is the camera mounted on the wall. The video capture interface uses V4L2 (video for Linux 2), which is an API interface for collecting multimedia information such as pictures, audio, and video under the Linux operating system platform. It is widely used in remote conferences and high-definition video surveillance in the fields of systems and embedded multimedia terminals. Among them, the Linux operating system is used by the majority of users because of its low cost, opensource network, code sharing, device independence, and good portability. The V4L2 interface assists in the collection of multimedia information such as pictures and videos by assisting the appropriate acquisition device and corresponding camera driver. The experimental video capture operating system model is shown in Figure 6. The device voltage requirements are shown in Table 3. 
TABLE 2: Comparison of storage rules.

\begin{tabular}{lccc}
\hline Feature & File storage & Graphics storage & Column storage \\
\hline Energy lasts & Year & Hour & Week \\
Number of nodes & 28 & 5 & 6500 \\
Reaction time & $7 \mathrm{~s}$ & $3 \mathrm{~s}$ & $25 \mathrm{~ms}$ \\
Effective range & $120 \mathrm{~m}$ & $10 \mathrm{~m}$ & $80 \mathrm{~m}$ \\
Data transfer rate & 12 & 2 & 200 \\
\hline
\end{tabular}

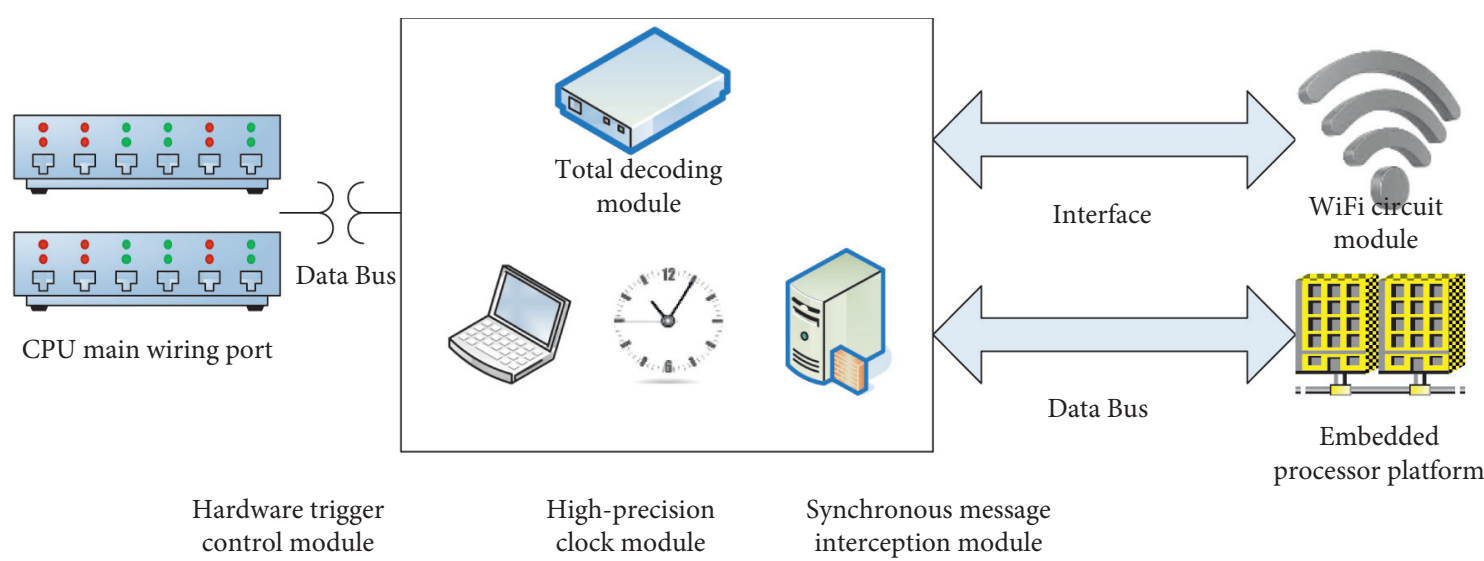

FIGURE 6: Video operating system model.

TABLE 3: Device voltage requirements.

\begin{tabular}{lcccc}
\hline Device name & ATI ES1000 & LCD screen & Embedded board & Power board \\
\hline Voltage & $4.2 \mathrm{~V} 1$ & $4 \mathrm{~V} 1$ & $5 \mathrm{~V} 1$ & $3.7 \mathrm{~V} 2$ \\
Device name & GeForce 9800 GTX & MAX4814 & DP9000 & DM47218 \\
Voltage & $3.3 \mathrm{~V} 2$ & $3.3 \mathrm{~V} 2$ & $-4 \mathrm{~V} 3.3$ & $-4 \mathrm{~V} 4$ \\
\hline
\end{tabular}

Experiment platform:

(1) Web server: HP ProLiant ILO 3

CPU: Intel E5620 $2.4 \mathrm{GHz}$

Memory: $2 \mathrm{~GB}$

Graphics card: ATI ES1000

Operating system: Windows Server 2003

Web service program: IIS6.0

(2) Client:

CPU: Intel Core Quad Q6600 2.4 GHz

Memory: $4 \mathrm{G}$ (practical $3.25 \mathrm{G}$ )

Graphics card: GeForce 9800 GTX

Operating system: Windows XP SP3

Browser: IE6.0

3.2. Cross-Compiler Environment Installation and Configuration. Cross-compilation can generate executable files that can be run by another platform on one platform, that is, programs compiled in a certain PC environment and code that can be run in another environment. This tool is a cross compiler. Because you cannot complete the required functions on the destination platform, such as compiling, or the target platform has no operating system, cannot run, and so on, you need the help of cross compiler. This article uses cross compilation on the Linux platform to generate executable files on the arm platform.

Cross-compiler tool version: arm-linux-gcc 4.4.3, compile platform: PC redhat 9 system.

\subsection{Evaluation Indicators}

3.3.1. Quality of Multimedia Services. Due to the adaptive media service approach, the code rate/quality is timevarying, and the received video is distorted relative to the original video. For video with the same content and encoding, different transmission adaptive algorithms usually get different video quality even if they are transmitted in the same network environment. The most intuitive way to evaluate the quality of a video service is the quality of the video at the receiving end, that is, comparing each corresponding pixel of the original reference video frame with the distorted video frame, thereby obtaining the degree of similarity or fidelity between the two. The simplest methods to measure fidelity are the mean square error (MSE) and peak signal to noise ratio (PSNR).

Mean Square Error (MSE):

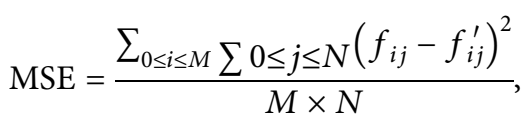


where $M, N$ represent the height and width of the video frame, respectively, and $f_{i j}$ and $f_{i j}^{\prime}$ represent the original reference video frame and the distorted video frame, respectively.

Peak signal-to-noise ratio (PSNR):

$$
\operatorname{PSNR}=10 \lg \frac{\left(2^{n}-1\right)^{2}}{\operatorname{MSE}^{\prime}},
$$

where $n$ is the number of bits per sample value. The PSNR is essentially the same as the MSE and is a logarithmic representation of the MSE. Based on the structural similarity (SSIM) of a single image, the quantized QoE is obtained by mapping the time-averaged SSIM onto the MOS. The mapping method is as follows:

$q^{\text {mos }}=13.3442 \log \left(1-q^{\text {ssim }}\right)+3.6226\left(1-q^{\text {ssim }}\right)+77.0117$.

$q^{\text {ssim }}$ is a time-averaged SSIM with a score from 0 to 100 , with 0 indicating the best QoE and 100 indicating the worst QoE.

3.3.2. Evaluation of Rate Control Algorithm. According to the DASH coding standard, the higher the code rate, and the higher the video quality, during video playback, the code rate is dynamically changing. Therefore, the video average bit rate can be used to measure the quality of the video. The average bit rate of a video is defined as

$$
\bar{B}=\frac{\sum_{n=1}^{N_{s}} B(n)}{N_{s}},
$$

where $B(n)$ represents the code rate of the video segment $n$.

The frame image quality can be measured by the average bit rate. Fluctuations in video quality can be measured by the frequency and magnitude of the quality switching. The frequency of rate switching is defined as

$$
f_{s}=\frac{S\left(N_{s}\right)}{N_{s} \cdot \tau}
$$

where $S\left(N_{s}\right)$ is the total number of handovers, where $\tau$ is the video duration.

The magnitude of the rate switch can be defined as

$$
a_{s}=\frac{\sum_{n=2}^{N_{s}}|B(n)-B(n-1)|}{N_{s} \cdot \tau} .
$$

It indicates the bit rate difference between two adjacent video segments.

\section{Results}

4.1. Multimedia Client Operating Platform. In order to ensure that the simulation is close to the actual system, we use Mininet as the simulation platform. Mininet is a lightweight soft-defined network R\&D and test platform that supports soft-defined network components such as Openflow and OpenvSwitch, supports complex topologies and custom topologies, and has good hardware portability (Limix compatibility). Use Mininet to virtualize a series of nodes, including hosts, switches, and controllers. The specific multimedia client platform interface is shown in Figure 7. At the same time, OpenvSwitch and POX were selected as virtual OpenFIow switches and controllers, respectively. The controller can configure the switch through the OF-Config interface. We implemented the controller program in Python, and the bandwidth of the link between network nodes was set to $1 \mathrm{Mbps}$. It can convert complex numerical calculations into matrix calculations, and the programming language has strong portability, high calculation efficiency, complete graphics processing capabilities, can realize the programmable characteristics of graphics, and has a rich toolbox.

The video used in the experiment is "Great Barrier Reef," which adopts SVC's spatial scalable coding method, including a basic layer and an enhancement layer with spatial resolutions of $320 \times 160$ and $640 \times 360$, respectively. The base layer has a code rate of $91 \mathrm{kbps}$ and a PSNR of $37.759 \mathrm{~dB}$ for the decoded video. Conversely, the enhancement layer has a code rate of $124 \mathrm{kbps}$ and a PSNR of $40.511 \mathrm{~dB}$ for the decoded video. In order to achieve multipath transmission, different video layers are sent through different UDP ports. In the experiment, the base layer occupies 50001 ports and the enhancement layer occupies 50002 ports. The switch matches the sending port in the forwarding table so that it can distinguish the video streams of different layers. Figure 8 shows the mobile multimedia client operation interface.

4.2. Sensing Application Analysis of Ubiquitous Power Network Structure. Based on the characteristics of the ubiquitous network that is guided by network applications, the information perception platform is combined with the application requirements of specific scenarios in the laboratory, and a sensor network application display platform based on the ubiquitous network architecture is developed to demonstrate the application under the ubiquitous network architecture. The characteristics and network characteristics of it are very meaningful. Analyze the characteristics of the ubiquitous network structure, as shown in Figure 9.

In the power system, the wireless sensor network can monitor, perceive, and collect various power parameters in the distribution area of the power network in real time and process this information to obtain more detailed and accurate information. The target bit rate comparison results of different algorithms optimized for the ubiquitous power sensor network tester are shown in Figure 10.

\subsection{Analysis of Rate Control Optimization Algorithm Based on} Multimedia Platform. The adaptive clustering routing algorithm can extend the network life cycle. In order to verify the relevant analysis conclusions of the algorithm design part, the network lifetime verification experiment designed in this paper is verified in the following aspects. The first set of control experiments set the statistics of the number of dead nodes, and the sampling was performed once every 50 rounds of simulation period. The simulation time was 500 rounds, and the unit packet size was 500 bits. The variation of the number of dead nodes in the network of a simulation 


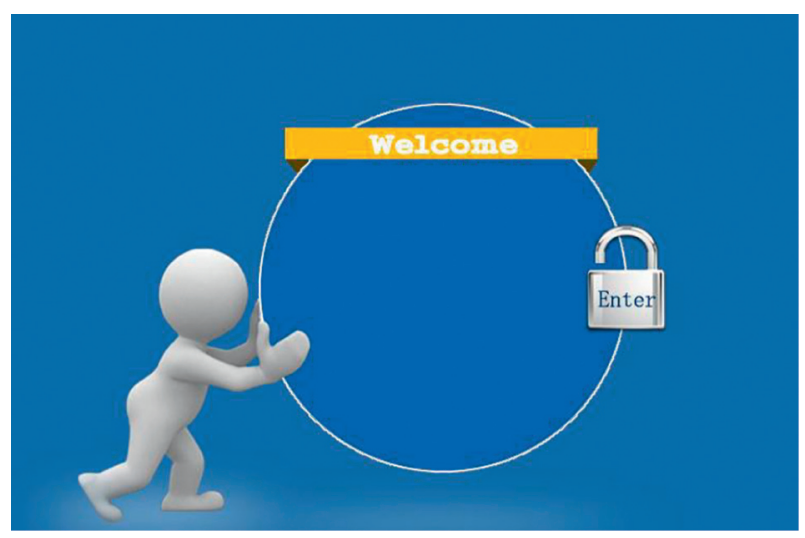

FIGURE 7: Multimedia system login interface.
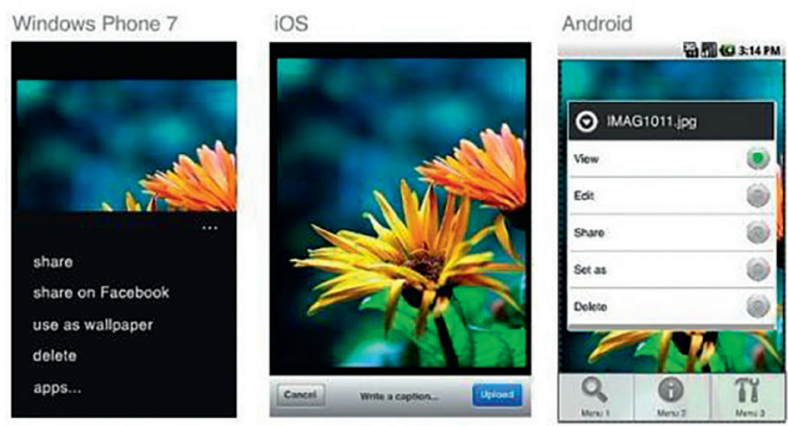

Figure 8: Mobile client interface.

process with the simulation time is studied. The statistical data curve is shown in Figure 11(a); the second group of controlled experiments changes each round of data packets under the premise of other conditions. Size, unit data packet variation range: $200-2000$ bits, ten sets of simulations, with the first node death time as the network lifetime, and the curve comparison of the network lifetime data is shown in Figure 11(b).

In order to verify the accuracy of the wireless link quality prediction model under the noise interference and multipath effect of the wireless communication link in different power industry environments, the article selects three scenarios of distributed photovoltaic power generation system, microgrid underground voltage conversion room, and energy storage equipment as the simulation experiment scenarios. It can be seen from Figure 11(a) that the network nodes using AODV and LEACH algorithm have half of the node energy exhaustion failure when the simulation time reaches 30 and 70 rounds, and the optimized adaptive family algorithm AC_AODV simulates the time when half of the nodes fail. After more than 100 rounds, as the simulation progresses, the number of failed nodes increases. At the same time, the AC-AODV algorithm has the least number of failed nodes, which indicates that the algorithm can make the network cluster head distribution more uniform, energy consumption is balanced, and there are more inter-clan hop routing mechanism can effectively save network energy consumption and extend the network lifetime. Figure 11(b) shows the comparison of the network lifetimes of different algorithms under ten sets of experiments after changing the unit packet size. It can be seen that as the unit packet size becomes larger, the energy consumed per round of packets is increased, and energy saving is achieved. Accelerated, the life cycle of the three algorithms is generally declining, but the AC-AODV algorithm is longer than the other two methods in different packet sizes. The results of multiple sets of algorithms show that the algorithm has good performance.

4.4. Comparison of Algorithms Based on Ubiquitous Power Sensor Networks. As shown in Table 4 and Figure 12, obviously, if collecting data on a single tower and sending it to the data center, it saves time compared to the mode of not collecting monitoring data. On the other hand, in long transmission lines, if you want to send monitoring data to the data center, it will pass many hops and the network mode will save more time. This shows the advantages of studying the sensor network model. Delay performance and energy costs are more reliable than the previously proposed security protocol network model. It can be proved that the ubiquitous power sensor network communication protocol designed by us can greatly reduce the energy consumption of the entire power long-distance transmission line, making the collection of monitoring information more energy-saving. This communication protocol will be beneficial to ubiquitous power. The wireless sensor around the sensor network is used because the communication protocol has a low demand for energy consumption, which is in line with the wireless sensor solar power supply. In terms of time delay, the communication protocol of this paper can also make the process of obtaining information in the data center faster and make the fault processing time faster.

After analysis, considering the limited CPU of the normal sensor node, because the normal sensor node can only perform some simple operation data collection, calculation, and transmission, so the GN has a powerful CPU and a separate power system so that the GN processing capability is stronger than NSN, GN performs the main protocol calculation steps, so we can solve the problem of low computing power of ordinary sensors.

\subsection{Performance Analysis of Simulation Network Algorithm} Presented by Client. Comparing the code rate change of the code rate control optimization algorithm, as shown in Figure 13, it can be found that even if the network fluctuates drastically, the video bit rate remains relatively stable. For example, compare the performance of the algorithm between $80 \mathrm{~s}$ and $100 \mathrm{~s}$. For the controller-side adaptive algorithm, the video bit rate is maintained at $6 \mathrm{Mbps}$. For the client's adaptive algorithm, the video bit rate is constantly shaking up and down between $5 \mathrm{Mbps} / \mathrm{s}$ and $6 \mathrm{Mbps}$. The jitter of the code rate will cause the video quality to flicker, which will reduce the quality of the experience.

This is also reflected in the performance indicators shown in Figure 14: the former video bit rate variation is $0.15 \mathrm{Mbps} / \mathrm{s}$; the frequency is $0.12 \mathrm{~s}$, lower than the latter $0.36 \mathrm{Mbps} / \mathrm{s}$ and $0.32 \mathrm{~s}$. This is because the rate control on the controller side is based on the congestion probability, 


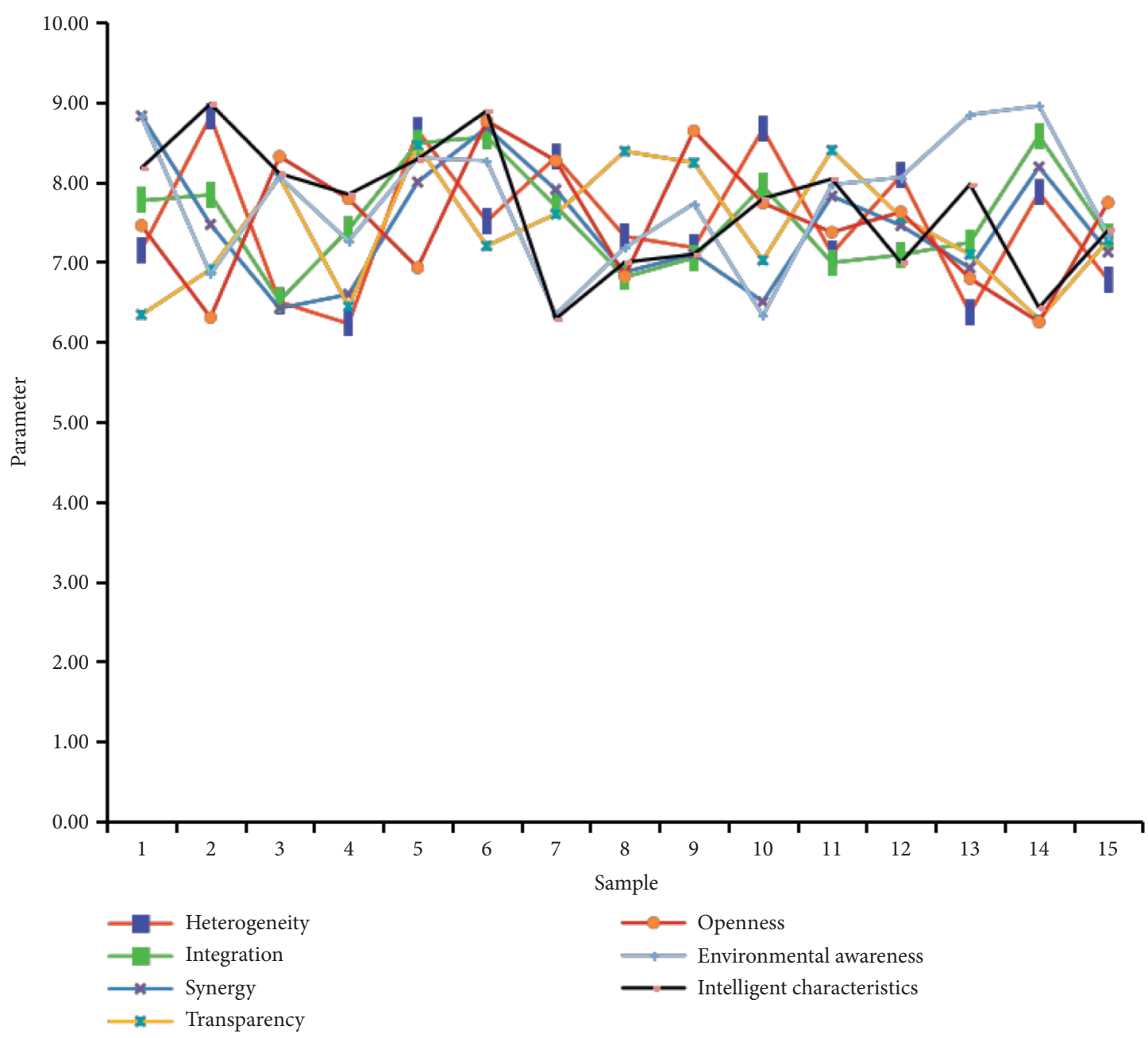

Figure 9: Characteristics of ubiquitous network structure.

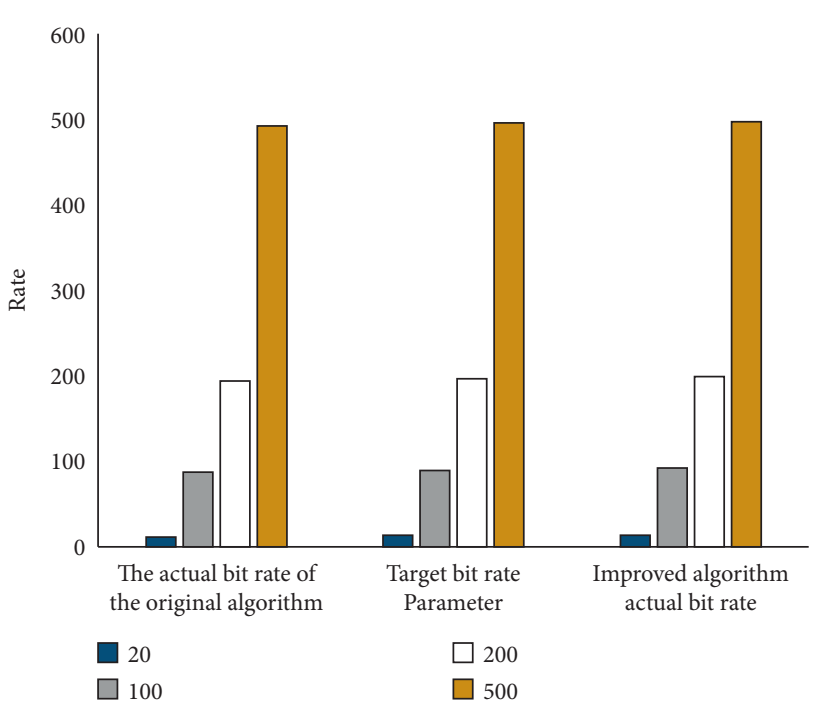

Figure 10: Comparison of target bit rates of different algorithms.

and the short-term changes of the network have little effect on the congestion probability. For the rate control of the client, the video bit rate depends on the instantaneous throughput of the network, so the fluctuation of the network will cause the rate jitter. The video frame format and other parameters are shown in Table 5.

Notify the online client, send a text message, and rotate the pan-tilt to the corresponding preset position for shooting and video recording. At the same time, the interface of the strategy library has buttons such as add, modify, delete, and save. You can add new strategies, modify and delete existing strategies, and save the set strategies. Evaluate the performance of the rate control optimization algorithm from the perspective of customer experience, and the results are shown in Figure 15.

\section{Discussion}

The multimedia operation platform based on NoSQL database and $\mathrm{B} / \mathrm{S}$ architecture can adaptively adjust the bit rate and routing of video according to the change of network conditions, thereby greatly improving the adaptive ability of video services to network changes. At the same time, due to the multipath transmission mode, the idle bandwidth resources on different paths are effectively utilized, and the video quality is improved. It can only locate the high-voltage 


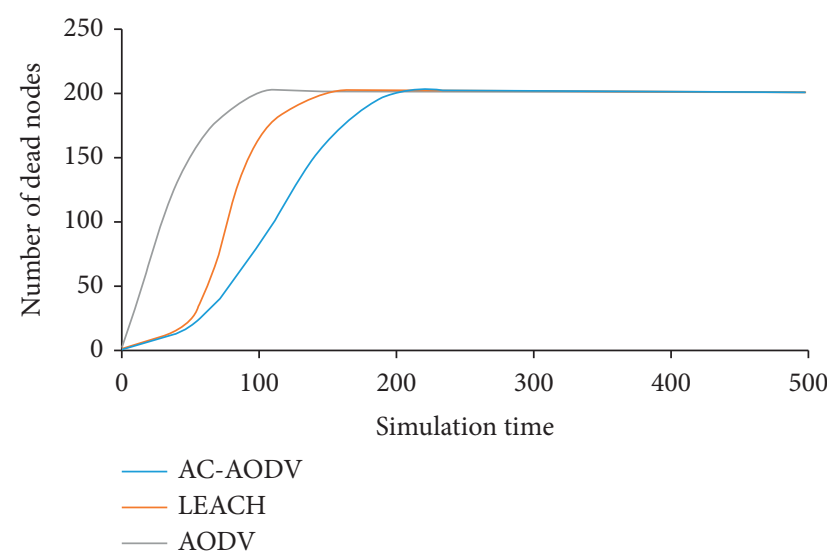

(a)

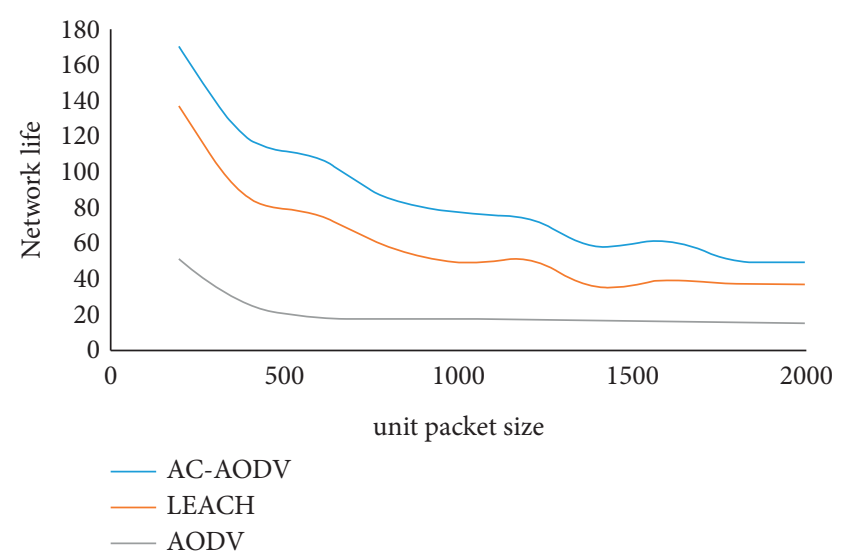

(b)

FIgURE 11: Network lifetime analysis chart.

TABLE 4: Comparison of delays with increasing hop count in different modes.

\begin{tabular}{lcc}
\hline Hop count & Time delay under power sensor network model & Time delay without power sensor network model \\
\hline$i=1$ & 35.01 & 36.12 \\
$i=2$ & 35.12 & 36.34 \\
$i=3$ & 35.61 & 35.98 \\
$i=4$ & 35.45 & 36.20 \\
$i=5$ & 35.34 & 36.07 \\
$i=6$ & 35.87 & 36.58 \\
$i=7$ & 36.31 & 37.89 \\
$i=8$ & 37.21 & 38.21 \\
\hline
\end{tabular}

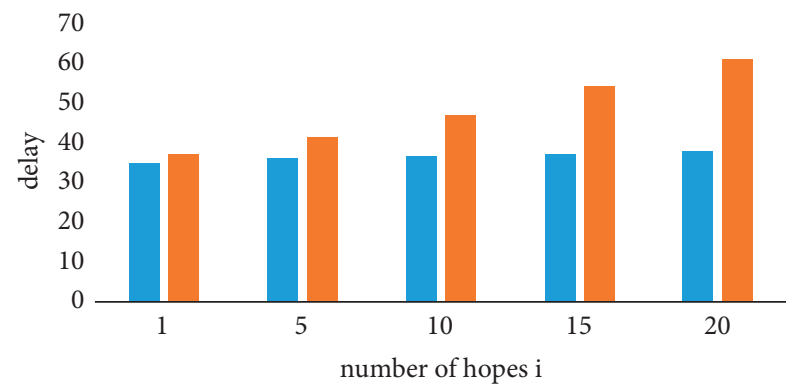

aN collect monitoring data from NSN then send to DC - NSN send monitoring data to DC

FIgURE 12: Time delay with increasing NSN.

nodes of the power line and can also obtain the data of the sensor network, realize the all-round monitoring of the power frame, and formulate its own behavior plan based on the data of the sensor network.

In the simulation, the player's startup delay is set to 333 milliseconds; that is, the video playback starts after 10 frames are cached. The underflow threshold of the video play queue length is 4 , that is, $\mathrm{QL}=4$. The parameters of the algorithm based on the underflow probability are set as follows: sliding window length $\mathrm{Sw}=80$; prediction window length $N=100$; forgetting factor $p=0.8$; and underflow probability thresholds $\mathrm{qu}=10-3$ and $\mathrm{qo}=10-8$. At the beginning of the experiment, only the base layer L01 video data is transmitted.

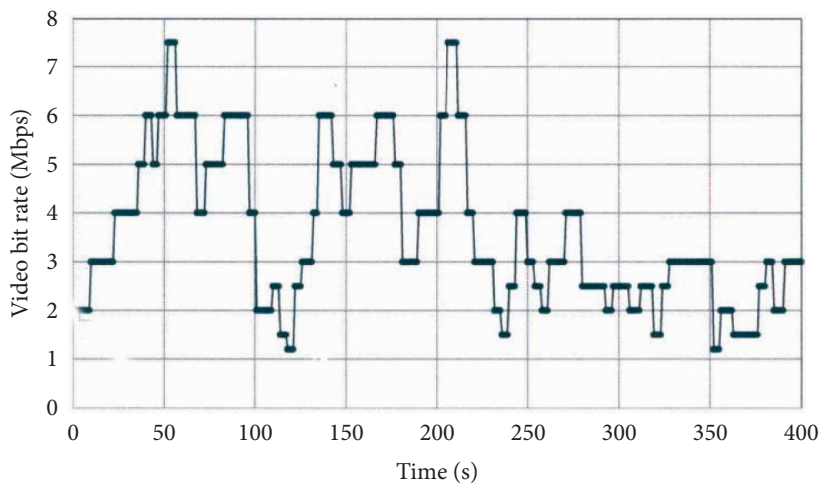

FIGURE 13: Code rate control optimization algorithm performance chart.

The proposed rate control optimization algorithm EBAODV of the ubiquitous power sensor network controls the broadcast direction of the route request by introducing a multiattribute energy balance mechanism in the route request phase so that the route bypasses the lower performance node, and secondly a link with a better link performance is selected as a data transmission link by a link performance function. The simulation results show that energy-balanced routing can effectively avoid the problem of uneven energy consumption in the network, improve the overall performance of the network, and extend the network life cycle.

Through the simulation attack, the specific response of the security protocol is analyzed to verify the security of the 


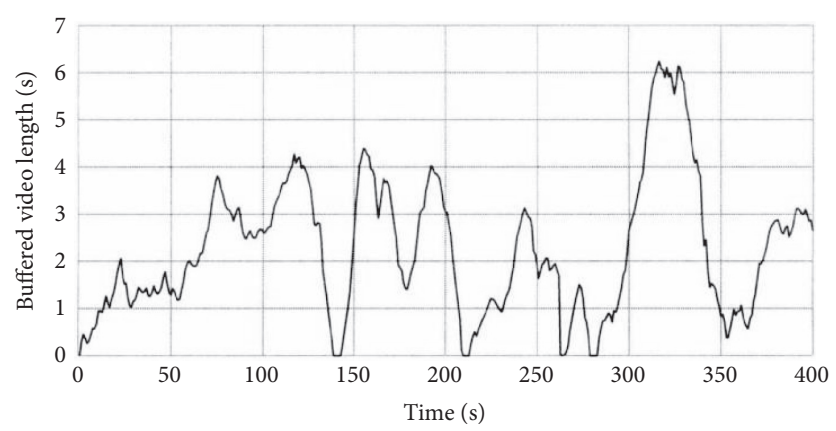

FIGURE 14: Client rendering effect changes.

TABle 5: Video frame format parameters.

\begin{tabular}{lccccc}
\hline Message & Preamble & Frame start flag & Video source address & Message data & Type of data \\
\hline Byte size & 8 & 2 & 7 & $0-2000$ & - \\
Data & $00 \ldots 00$ & $0 * 01$ & - & - & $0 * 0400$ \\
\hline
\end{tabular}

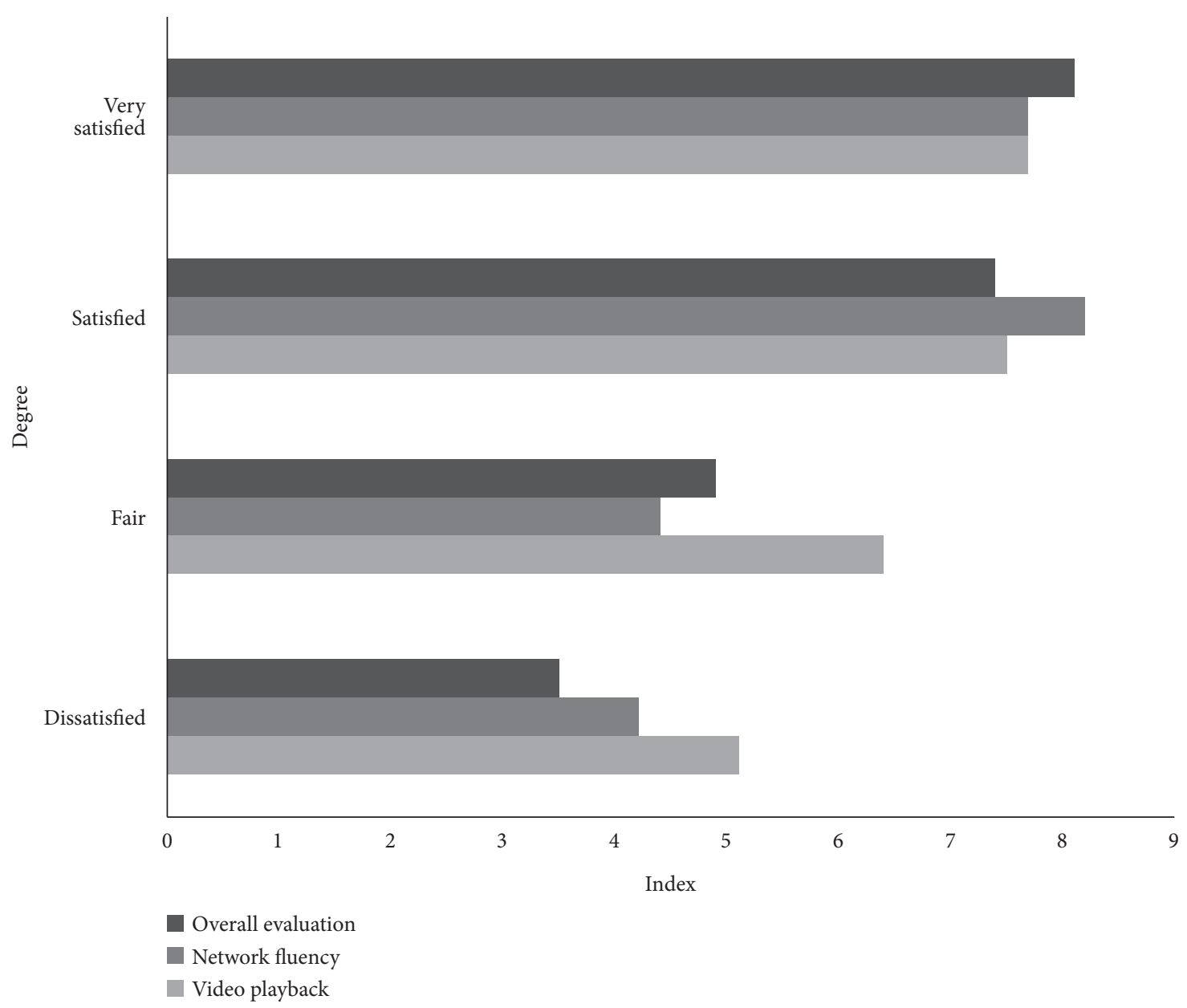

FIgURE 15: Customer experience evaluation results.

protocol, and the designed communication protocol is safe and reliable. By calculating the energy consumption analysis, the low energy consumption of the communication protocol is verified, and the delay of the power tower wireless sensor network is compared with the delay of the common smart grid communication. It is concluded that the construction of the communication model can reduce the delay and thus prove superiority of the design of this paper. 


\section{Conclusion}

In this paper, the rate control optimization algorithm for multimedia-based ubiquitous power sensor network tester is studied from three aspects: constructing algorithm verification platform based on multimedia technology, performance analysis, and ubiquity of rate control optimization algorithm based on multimedia technology. Performance analysis of power sensor networks is done. In order to overcome the randomness, complexity, and time-varying of the network, high-definition and smooth multimedia services are provided, and the problem is optimized and modeled by stochastic optimization. Experiments show that the algorithm can improve the video quality as much as possible while ensuring smooth playback. The specific work is summarized as follows:

(1) Establish an algorithm verification platform based on multimedia technology. This article uses a scalable video coding method to design a client-driven scalable video transmission system. The underflow probability is proposed and defined considering the state of the channel and the buffer state at the receiving end. The impact of changes in channel quality on video playback can be predicted by estimating the underflow probability. In order to verify the feasibility of the actual deployment of the algorithm, the prototype system is designed and verified. Finally, simulation experiments show that the algorithm can effectively improve the video quality.

(2) Propose a rate control optimization algorithm based on multimedia technology. In order to ensure the reliability of network communication, a reliable multipath routing algorithm based on AODV is proposed. Based on the energy equalization algorithm, a node-independent routing mechanism and a link quality estimation model are introduced to establish an independent and reliable active/standby communication chain to the destination node. With this reliable link redundancy mechanism, reliable data transmission is achieved.

(3) Under the Linux platform, a large number of tests are carried out, and the performance of the control code rate is analyzed. It is concluded that $A B R$ is the most suitable rate control algorithm for video transmission. According to the CQP experiment results, the $\mathrm{QP}$ value set in the improved ABR algorithm is up to 40. Compared with the previous algorithm, according to the experimental results, it is closer to the target code rate, more accurate, and more suitable for application in real-time video transmission systems.

(4) Verifying the security from the construction of the ubiquitous power sensor network to the final monitoring data collection and upload process by simulating hypothetical attacks and failures. By analyzing and comparing the energy consumption and delay of constructing a ubiquitous power sensor network and not constructing a ubiquitous power sensor network, it is proved that building a ubiquitous power sensor network can reduce the energy consumption and delay of monitoring data collection and verify the superiority of building a ubiquitous power sensor network.

\section{Data Availability}

This article does not cover data research. No data were used to support this study.

\section{Conflicts of Interest}

The authors declare that they have no conflicts of interest regarding the publication of this paper.

\section{Acknowledgments}

This work was supported by the State Grid Corporation Science and Technology Project (Research on comprehensive calibration platform and remote calibration key technology of special equipment for power equipment testing based on "Internet + ").

\section{References}

[1] B.-K. Park, H.-W. Kim, J. H. Park, and Y.-S. Jeong, “Adaptive power management scheme using many-core for maximizing network topology lifetime based on ubiquitous computing," Journal of Systems Architecture, vol. 77, pp. 63-71, 2017.

[2] P. Gope, "Enhanced secure mutual authentication and key agreement scheme with user anonymity in ubiquitous global mobility networks," Journal of Information Security and Applications, vol. 35, pp. 160-167, 2017.

[3] J. Santana, D. Marrero, E. Macías, V. Mena, and Á. Suárez, "Interference effects redress over power-efficient wirelessfriendly mesh networks for ubiquitous sensor communications across smart cities," Sensors, vol. 17, no. 7, p. 1678, 2017.

[4] G. Fan, "The ubiquitous network era, the three development trends of smart residential areas," Intelligent Building and Smart City, vol. 17, no. 9, 2018.

[5] J. Xing, "The enlightenment of college English maker space construction based on ubiquitous network learning platform," Contemporary Educational Practice \& Teaching Research, no. 7, pp. 44-45, 2018.

[6] J. Chai and X. Xu, "Study on the construction of wisdom workshop based on ubiquitous network technology," China Education Technique and Equipment, no. 20, pp. 19-21, 2018.

[7] P. Ni, W. Wang, and J. Ni, "Study on the security policy architecture of digital library under the ubiquitous network environment," Journal of Library and Information Science, vol. 36, no. 4, pp. 10-16, 2018.

[8] N. Wang, "Research on information organization mechanism in ubiquitous network," Modern intelligence, vol. 38, no. 5, pp. 25-31+36, 2018.

[9] Y. Duan and Y. Liu, "Study on the maturity of government information service in ubiquitous network environment," Information Science, vol. 36, no. 7, pp. 36-39, 2018.

[10] X. Pan and Y. Chen, "Inquiring into the intelligent service mode of public libraries in China under the ubiquitous network environment," Information Science, vol. 36, no. 5, pp. 30-34, 2018. 
[11] J. Jiao, "Research on the construction of experimental teaching platform based on ubiquitous network," Internet of Things Technology, vol. 6, no. 11, pp. 117-118, 2016.

[12] Q. Zhang, "Design of knowledge fusion model for ubiquitous networks," Journal of Shaoguan University, vol. 38, no. 3, pp. 21-24, 2017.

[13] Zhuoma, "Pan-network construction and the construction of smart communities in Mengdong area," Ethnic Forum, no. 3, pp. 96-99, 2017.

[14] A. Etesaminia and A. H. Mazinan, "An efficient rate control algorithm for JPEG2000 based on reverse order," Journal of Central South University, vol. 24, no. 6, pp. 1396-1405, 2017.

[15] M. H. Park, J. Y. Choi, and J. K. Choi, "A network-aware encoding rate control algorithm for real-time up-streaming video services," IEEE Communications Letters, vol. 21, no. 7, pp. 1653-1656, 2017.

[16] S. K. Lakshmi, C. Arun, and F. C. Dpra, "Dynamic probabilistic rate allocation and flow control algorithm during congestion in wireless sensor network," Journal of Computational and Theoretical Nanoscience, vol. 14, no. 9, pp. 4268-4277, 2017.

[17] H. Guo, H. Luo, S. Liu, N. Lin, and B. Yang, "An improved R- $\lambda$ model rate control algorithm," Computer Science, vol. 46, no. 3, pp. 142-147, 2019.

[18] H. Guo and S. Liu, "A HEVC low delay coding rate control algorithm," Computer Applications and Software, vol. 36, no. 3, pp. 162-167+191, 2019.

[19] T. Yan, J. Huo, Z. Lei, and D. Chen, "The MV-HEVC rate control algorithm based on similarity analysis," Journal of Fuzhou University (Natural Science Edition), vol. 47, no. 1, pp. 18-23, 2019.

[20] W. Zhan, J. Xiao, Y. Chen, and J. Chen, "Rate control algorithm for adaptive rate video live broadcasting," Computer Engineering, vol. 45, no. 3, pp. 268-272, 2019.

[21] H.-z. Tang, N. Guo, and C. Ying-tao, "HEVC frame layer rate control algorithm based on content complexity," Journal of Xi'an University of Posts and Telecommunications, vol. 22, no. 6, pp. 98-103, 2017.

[22] H. Liu, L. Liu, H. Luguo, and J. Liao, "A HEVC rate control algorithm based on scene switching," Tv Engineering, vol. 41, no. 6, pp. 1-5, 2017.

[23] W. Chen, F. Chen, D. Jin, J. Li, and Z. Peng, "The HEVC intraframe rate control algorithm based on complexity analysis," Computer Applications and Software, vol. 34, no. 01, pp. 143-148+174, 2017.

[24] B. Zheng, C. Yang, and W. Ou, "Study on DVC code rate control algorithm for extrapolation structure without feedback," Computer Engineering and Applications, vol. 53, no. 01, pp. 207-212, 2017.

[25] Q. Rong and J. Yang, "Improvement of LCU layer rate control algorithm based on HEVC," Computer Applications and Software, vol. 33, no. 5, pp. 136-139, 2016. 